University of Wollongong

Research Online

Faculty of Engineering - Papers (Archive)

Faculty of Engineering and Information

Sciences

2005

\title{
Edge-on face-to-face MOSFET for synchrotron microbeam dosimetry: MC modeling
}

Anatoly B. Rosenfeld

University of Wollongong, anatoly@uow.edu.au

E. A. Siegbahn

European Synchrotron Radiation Facility, France

E. Brauer-Krisch

European Synchrotron Radiation Facility, France

A. Holmes-Siedle

REM Oxford Ltd, UK

M. L. Lerch

University of Wollongong, mlerch@uow.edu.au

See next page for additional authors

Follow this and additional works at: https://ro.uow.edu.au/engpapers

Part of the Engineering Commons

https://ro.uow.edu.au/engpapers/77

\section{Recommended Citation}

Rosenfeld, Anatoly B.; Siegbahn, E. A.; Brauer-Krisch, E.; Holmes-Siedle, A.; Lerch, M. L.; Bravin, A.; Cornelius, I.; Takacs, G. J.; Painuly, N.; Nettleback, H.; and Kron, T.: Edge-on face-to-face MOSFET for synchrotron microbeam dosimetry: MC modeling 2005.

https://ro.uow.edu.au/engpapers/77

Research Online is the open access institutional repository for the University of Wollongong. For further information contact the UOW Library: research-pubs@uow.edu.au 


\section{Authors}

Anatoly B. Rosenfeld, E. A. Siegbahn, E. Brauer-Krisch, A. Holmes-Siedle, M. L. Lerch, A. Bravin, I. Cornelius, G. J. Takacs, N. Painuly, H. Nettleback, and T. Kron 


\title{
Edge-on Face-to-Face MOSFET for Synchrotron Microbeam Dosimetry: MC Modeling
}

\author{
Anatoly B. Rosenfeld, Senior Member, IEEE, Erik A. Siegbahn, Elke Brauer-Krish, \\ Andrew Holmes-Siedle, Senior Member, IEEE, Michael L. F. Lerch, Member, IEEE, Alberto Bravin, \\ Iwan M. Cornelius, George J. Takacs, Nirmal Painuly, Heidi Nettelback, and Tomas Kron
}

\begin{abstract}
The dosimetry of X-ray microbeams using MOSFETs results in an asymmetrical beam profile due to a lack of lateral charged particle equilibrium. Monte Carlo simulations were carried out using PENELOPE and GEANT4 codes to study this effect and a MOSFET on a micropositioner was scanned in the microbeam. Based on the simulations a new method of microbeam dosimetry is proposed. The proposed edge-on face-to-face (EOFF) MOSFET detector, a die arrangement proposed here for the first time, should alleviate the asymmetry. Further improvement is possible by thinning the silicon body of the MOSFET.
\end{abstract}

Index Terms-Charged-particle equilibrium (CPE), dose enhancement effects (DEEs), dosimetry, microbeam, MOSFET, radiotherapy, synchrotron.

\section{INTRODUCTION}

$\mathbf{X}$ -RAY beams have been important tools in medicine, imaging, and radiation testing for many years Indeed, the scanning of a small MOSFET chip to map the "broad" beam spot of an X-ray tube in 1970 provided the first data showing that MOSFETs were suitable for use as dosimeters [1]. Other authors went further and used small beams to determine the particular elemental structure responsible for the failure of a device [2]. The development of synchrotron light sources with intensity of X-rays six orders of magnitude higher than conventional X-ray tubes has introduced unique experimental techniques for radiation testing of single microstructures-cells and devices - by the local deposition of dose using microbeams of micron size [2]. Another unique application of synchrotron $\mathrm{X}$-ray microbeams is microbeam radiation therapy (MRT): a new radiation oncology modality that is being developed at the European Synchrotron Radiation Facility (ESRF) and Brookhaven National Laboratory (BNL). The measurement of intensity distribution and dose pattern delivered to the sensitive elements of microelectronic devices or tissue is a challenge due to the relatively large size of conventional detectors.

MOSFET dosimetry has an advantage due to the extremely small size of the dosimetric volume, namely the gate oxide of

Manuscript received August 26, 2005; revised September 1, 2005.

A. B. Rosenfeld, M. L. F. Lerch, I. M. Cornelius, G. J. Takacs, N. Painuly, and H. Nettelback are with the Centre for Medical Radiation Physics, University of Wollongong, Wollongong NSW 2522, Australia (e-mail: anatoly@uow.edu.au).

E. A. Siegbahn, E. Brauer-Krish, and A. Bravin are with the European Synchrotron Radiation Facility, 38043 Grenoble Cedex, France.

A. Holmes-Siedle is with REM, Oxford, Ltd., Eynsham, Witney, OX29 4PD England, U.K.

T. Kron is with the Peter MacCallum Cancer Institute, Melbourne, Victoria 8006, Australia.

Digital Object Identifier 10.1109/TNS.2005.860704 the transistor. The size of the microbeam we use is similar to the geometrical area of the gate of the MOSFET in the plane of the silicon substrate [3], [4]. Some of the present authors proposed a new approach for dosimetry with high spatial resolution in synchrotron microbeams: the "edge-on (EO) MOSFET." The EO mode was successfully tested at ESRF and BNL [5]-[8]. The spatial resolution is limited in theory only by the thickness of the gate oxide. However, other factors interfere with the accuracy, especially the lack of local charged-particle equilibrium (CPE) and dose enhancement related to the design of the chip. A better understanding of such effects are particularly important for dosimetry of the multiple microbeam arrays used for MRT, for which absolute dosimetry and correct tissue dose profile measurements are vital to the preservation of healthy tissue and the killing of tumor tissue.

The response of the MOSFET to a wide energy range of photons in broad beams has been studied in wide radiation beams, mostly in free air geometry [9]-[12]. Severe dose enhancement effects (DEEs) arise from high $\mathrm{Z}$ metal $(\mathrm{Au}, \mathrm{Ni}, \mathrm{Cu}$ ) overlayers near the oxide gate. These materials attenuate photons and increase the number of secondary electrons crossing the gate oxide, especially at low photon energies. A lack of electronic equilibrium at the Metal-Si or $\mathrm{Si}-\mathrm{SiO}_{2}$ interfaces, with thicknesses less than the range of the secondary electrons, affects the response of a MOSFET at high photon energies. The dose enhancement factor (DEF), the ratio of the average dose to the sensitive region with respect to the dose at true CPE, was investigated for many designs of MOSFET in different photon fields. The DEF for a bare chip in a wide X-ray beam (for which the dimensions of the beam are greater than the entire dosimeter) depends on the direction of the photon beam and is more pronounced for low energy X-rays [13]. For packaged MOSFET devices in standard carriers like TO-18, TO-5, and dual in line (DIL), the DEF is not monotonic with photon energy. This is related to backscattering of electrons from high $\mathrm{Z}$ components of the packaging [14].

In contrast to the broad X-ray beam in free air, the response of a MOSFET in a water phantom is driven by secondary electrons from the surrounding medium rather than direct interaction of photons with the gate oxide. This is determined by the Bragg-Gray cavity theory:

$$
\frac{D_{\mathrm{SiO}_{2}}}{D_{\text {water }}}=\frac{S_{\mathrm{SiO}_{2}}}{S_{\text {water }}}
$$

Where $D$ is the dose response of the device or tissue, and $S$ is the stopping power of electrons in a particular medium (the 
DEE due to the overlayers is ignored). The ratio of the electron stopping powers is constant over a wide range of electron energy. However, even in the case of low energy X-rays, where the phantom is acting only as an attenuation medium for photons, the response will be driven by the material immediately on top of the gate. Hence, in water-based dosimetry the DEF can introduce an error. To give an example, the response of a gate oxide on a 500 micron deep silicon substrate, irradiated in water from the silicon direction, will be driven by silicon for all photons of spectra with energy lower than about $400 \mathrm{keV}$. For photon energies less then 15-20 keV, the DEF is high and special attention should be paid to the design and calibration of MOSFET dosimeters for low energy X-ray applications [for computer tomography (CT) scanners and mammography units].

The aim of this paper is to understand the response and suggest improved design features of a MOSFET used in the EO mode for synchrotron microbeam scanning. The added complexity in comparison with broad beam applications arises from the requirement for mapping to one micron (or better) spatial resolution in water. Changing the lateral electronic disequilibrium during the scanning across the microbeam is unpredictable and so it is a challenge to achieve the requirement.

We present Monte Carlo simulations of the response of an EO MOSFET dosimeter on an ESRF synchrotron beam. We require a solution to the poor CPE expected for single MOSFETs. The one which we propose at the end of this paper is called the edge-on face-to-face (EOFF) MOSFET.

\section{EXPERIMENTAL Details AND Simulation Technique}

\section{A. Synchrotron X-Ray Source for MRT}

MRT is carried out on ESRF's Medical Beamline, ID17. The Synchrotron Radiation X-ray beam is produced by bunches of $6 \mathrm{GeV}$ electrons circulating in an 844 meter circumference, evacuated ring with an orbit time of 2.82 microseconds. The maximum ring current of $200 \mathrm{~mA}$ has a typical decay time of 50 hours. The source of radiation is a 21 pole, $150 \mathrm{~mm}$ period wiggler magnet in the storage ring which has a $1.5 \mathrm{~T}$ magnetic field. The average energy of the X-rays produced is $100 \mathrm{keV}$ [8].

For our measurements, the maximum current was $20 \mathrm{~mA}$ with a typical lifetime of 6 hours. The beam in the first hutch of the ID17 beamline is spatially fractionated into microbeams at a distance of $33 \mathrm{~m}$ from the wiggler source using a multislit collimator allowing $500 \mu \mathrm{m}$ high and (25-50) $\mu \mathrm{m}$ wide microbeams with changeable pitch of up to $400 \mu \mathrm{m}$. The number of the microbeams is 70-100. A set of tungsten slits of various sizes can be installed on a three-axis stage behind the collimator, either to shield from scattered radiation (vertical height of the slits $=500 \mu \mathrm{m}$ ) or to select the desired microbeam(s) (25 $\mu \mathrm{m}$ horizontal opening size in case of single beam selection). The EO MOSFET detector embedded in a PMMA (Polymethyl Methacrylate) phantom was installed on the computer controlled sample stage (three-axis goniometer) $1.00 \mathrm{~m}$ downstream from the collimator allowing stepping of $0.5 \mu \mathrm{m}$. An ionization chamber at the far end of the hutch is used for alignment. Lasers, gafchromic films, and slits were used to align the MOSFET with the microbeam.

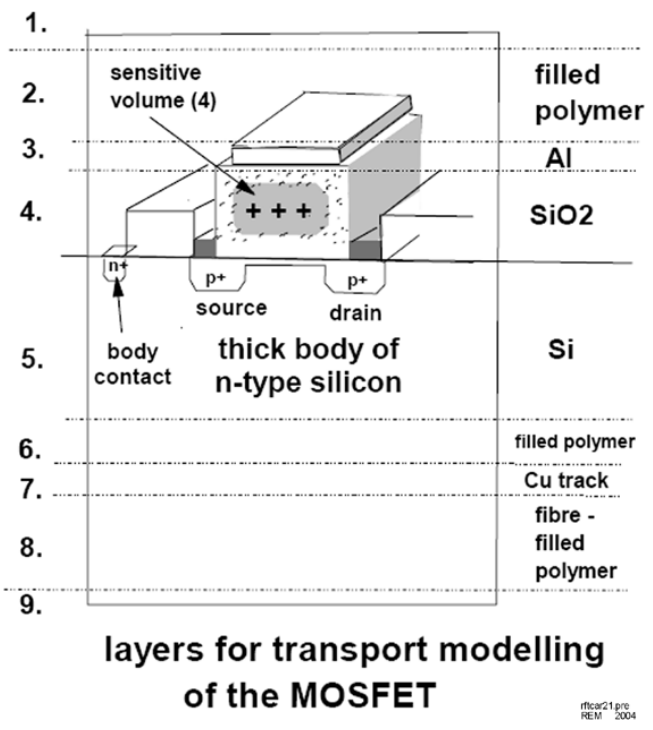

Fig. 1. Metal-oxide-silicon field-effect structure employed in the REM TOT series of dosimeters (RADFETs).

A fast shutter system is used on the beam to adjust the dose to the object. The highly reproducible minimum opening time of ID17's fast shutter is $10 \mathrm{~ms}$. Exact opening time is measured by a radiation-shielded photodiode which is connected to an oscilloscope at the end of the beam path.

The critical parameter in MRT technology responsible for the quality of radiation treatment is a dose pattern in tissue characterized by the peak-valley dose ratio which is dependent on the dose distribution from each single beam. Characterization of this dose pattern produced by a single beam is essential for the success of MRT.

\section{B. Geometry and Composition of the MOSFET Probe}

Our MOSFET real time dosimetry instrumentation for MRT experiments has previously employed the REM TOT 500 quadruple MOSFET detector. An ESM photo of topology of that chip can be seen in [8]. Two of the FETs have an oxide thickness of about $1 \mu \mathrm{m}$ and another two of about $0.16 \mu \mathrm{m}$. The Si body of the MOSFET is about $500 \mu \mathrm{m} \times 1000 \mu \mathrm{m} \times 1000 \mu \mathrm{m}$. The MOSFET chip was attached to a KAPTON pigtail containing $\mathrm{Cu}$ pads for placement of the chip and wire bonding. A filled epoxy globule covers the MOSFET chip. A new structure, the REM TOT 600, is now being introduced into the work. A generalized cross section of both types is given in Fig. 1. For simulation, we split the structure into layers. The outermost layers, 1 and 9, represent the surrounding medium; in our case a PMMA phantom. The following layers in Fig. 1 are parts of the sensor itself:

2. Encapsulant. Silica-filled black polymer of thickness about $0.5 \mathrm{~mm}$. Composite material: epoxy resin [diglycidyletherbisphenolA (DGEBA)] percentage w/w 90pc composition $\mathrm{C}_{18} \mathrm{H}_{18} \mathrm{O}_{3}$, silica powder [Cabot Cab-o-sil] percentage w/w 8 pc composition $\mathrm{SiO}_{2}$, carbon powder percentage $\mathrm{w} / \mathrm{w} 2 \mathrm{pc}$.

3. Gate. Al electrode with thickness $1 \mu \mathrm{m}$

4. Gate dielectric. Thermally oxidized silicon; the sensitive dosimetric volume of the MOSFET detector. 
5. Body of the MOSFET chip is always crystalline silicon; the thickness of the wafer is variable by lapping but, for the REM product, it is $0.5 \mathrm{~mm}$.

6. Die bond. Silica-filled black polymer adhesive with a thickness of about $0.1 \mathrm{~mm}$. The components are as for layer 2 .

7. Tracks. Etched or printed circuit tracks, plated on top with $\mathrm{Ni}$ and $\mathrm{Au}$. Cu $17 \mu \mathrm{m}$, Ni $10 \mu \mathrm{m}$, Au $1 \mu \mathrm{m}$.

8. Carrier. Glass-fiber reinforced polymer carrier with or without a Kapton layer. For the experiments described, layer 8 included a $0.1 \mathrm{~mm}$ Kapton flat flexible cable of dimensions $5 \mathrm{~mm} \times 60 \mathrm{~mm}$ lying above a fiber-filled polymer stiffener measuring $0.3 \mathrm{~mm} \times 6 \mathrm{~mm} \times 8 \mathrm{~mm}$.

The chip is completely encapsulated by a silica-filled epoxy globule and so scattering from bonding wires is not expected at the relevant energies. In the general case all layers are important for the simulation of the deposited dose in the gate of the MOSFET dosimeters as they can attenuate or enhance dose. As for the surface geometry of the MOSFET mask, simulation of a 3D model of the MOSFET dosimeter including the gate shape would be a complicated task. A simplified model was introduced, reflecting the relevant geometry of the microbeam relative to the MOSFET chip and the physics of the interaction of low energy X-rays for this geometry. The TOT600 die, to be used in the next phase, is designed to make simulation of the gate region much simpler and its accuracy in microbeam dosimetry more precise.

\section{Simulation Model}

MC simulations of deposited doses in the gate oxide by previous workers dealt with broad beams in free-air geometry, or in a solid water phantom [15], and mainly with the beam perpendicular to the surface ("front on"). Results agree well with experimental data. In the present paper we are simulating the response of the EO MOSFET detector, beam parallel to the surface and embedded in a PMMA phantom and irradiated by a synchrotron beam with cross section of only $25 \mu \mathrm{m} \times 500 \mu \mathrm{m}$. The key difference is that the smaller dimension of that beam is much less than that of the scattering layers, that is, the Si body and epoxy globule. The direction of the microbeam means that the DEE are related to laterally scattered electrons and lateral electronic disequilibrium. By contrast, a vertically incident broad beam generates secondary electrons mostly in the beam direction (at least for high energy photons). Taking into account the photon spectrum of the synchrotron X-ray beam with average energy $100 \mathrm{keV}$ it is clear that the CPE of the MOSFET is driven by the $\mathrm{Si}$ body and black epoxy (the range of secondary $100 \mathrm{keV}$ electrons is only $80 \mu \mathrm{m}$ in $\mathrm{Si}$ ). The degree of DEE from the secondary electrons changes during scanning of the EO MOSFET across the microbeam. Increasing lateral disequilibrium occurs as the proportion of the microbeam in layer 2 increases. Earlier studies of broad beams using conventional ICs had no need to address this problem.

Fig. 2 shows two views of a model structure for the EO MOSFET detector and geometry of the microbeam. The scanning direction is along the $Y$-axis. The $\mathrm{Si}$ body is $1 \mathrm{~mm} \times 1 \mathrm{~mm} \times 0.5 \mathrm{~mm}$. The active volume is the $\mathrm{SiO}_{2}$ layer
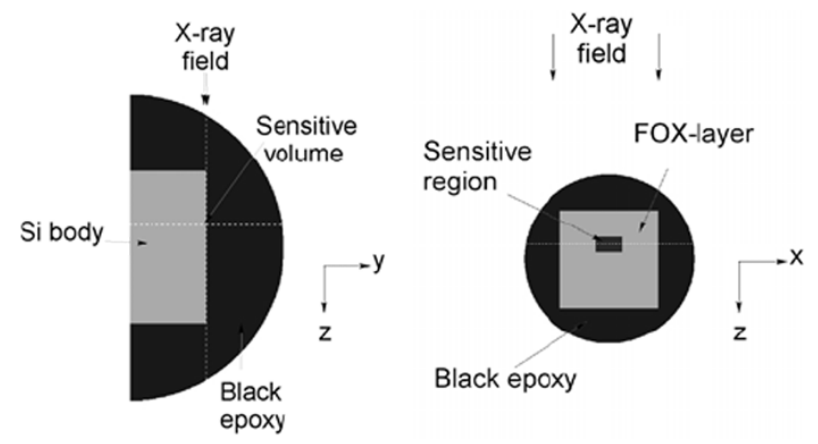

Fig. 2. EO MOSFET model for Monte Carlo simulations (not to scale). The limits and the direction of the X-ray field are indicated by arrows: (left) a vertical section in the $z y$ plane of the laboratory detector system; (right) a horizontal section in the $x z$ plane of the laboratory detector system.

on its top surface. The active $\mathrm{SiO}_{2}$ volume is only about $270 \times \times 180 \mu \mathrm{m}^{3}$. A similar but inactive layer of "field oxide (FOX)," visible in Fig. 2, completely surrounds it. Al layers above the drain, source, etc., and details of the KAPTON substrate (layers 6-8, Fig. 1) were omitted as they contribute no secondary electrons for the single microbeam under consideration. The oxide charging process is simplified; electron-hole recombination in the gate oxide, related to dense plasma tracks from low energy electrons ionizing the gate oxide [6], have been ignored. This will be considered in future papers.

For simulation, a single, narrow planar (rectangular) beam was considered. The MOSFET lay inside a PMMA cube with side-length $10 \mathrm{~cm}$. The MOSFET was moved after each simulation in a direction perpendicular to the direction of the beam, giving the dose profile. Phantom dimensions and materials were the same as for earlier studies [8].

The X-ray energies sampled from the measured spectrum at beamline ID17 are presented in Fig. 3. Photon energies are from $30 \mathrm{keV}$ to $600 \mathrm{keV}$. The probability of having a photon energy above $300 \mathrm{keV}$ is seen to be small. The height of the planar irradiation field was $500 \mu \mathrm{m}$ and the width $25 \mu \mathrm{m}$. In order to facilitate comparisons with other studies, the small divergence of the beam has been ignored in the simulations for this study. The collimators and slits have not been incorporated into the simulation geometry and all photons are assumed to start on top of the phantom with a common direction opposite to the normal of the phantom surface. Simulations were carried out using Monte Carlo codes PENELOPE and GEANT4.

\section{PENELOPE Monte Carlo Code}

PENELOPE is a general-purpose MC simulation package developed at the University of Barcelona [16]. The developers of this code have put special emphasis on the implementation of accurate low-energy electron/photon cross sections. In this work the 2003 version of the code was used.

For photons, the interaction cross sections for coherent, photoelectric and Compton scattering, all relevant for the MRT application, are implemented in the code. Compton scattering is the dominant photon interaction in low-atomic-number materials of interest in radiation therapy, for the X-ray spectrum generated by the ESRF wiggler. When simulating Compton interactions, as corrections to the normal Klein-Nishina formula, 


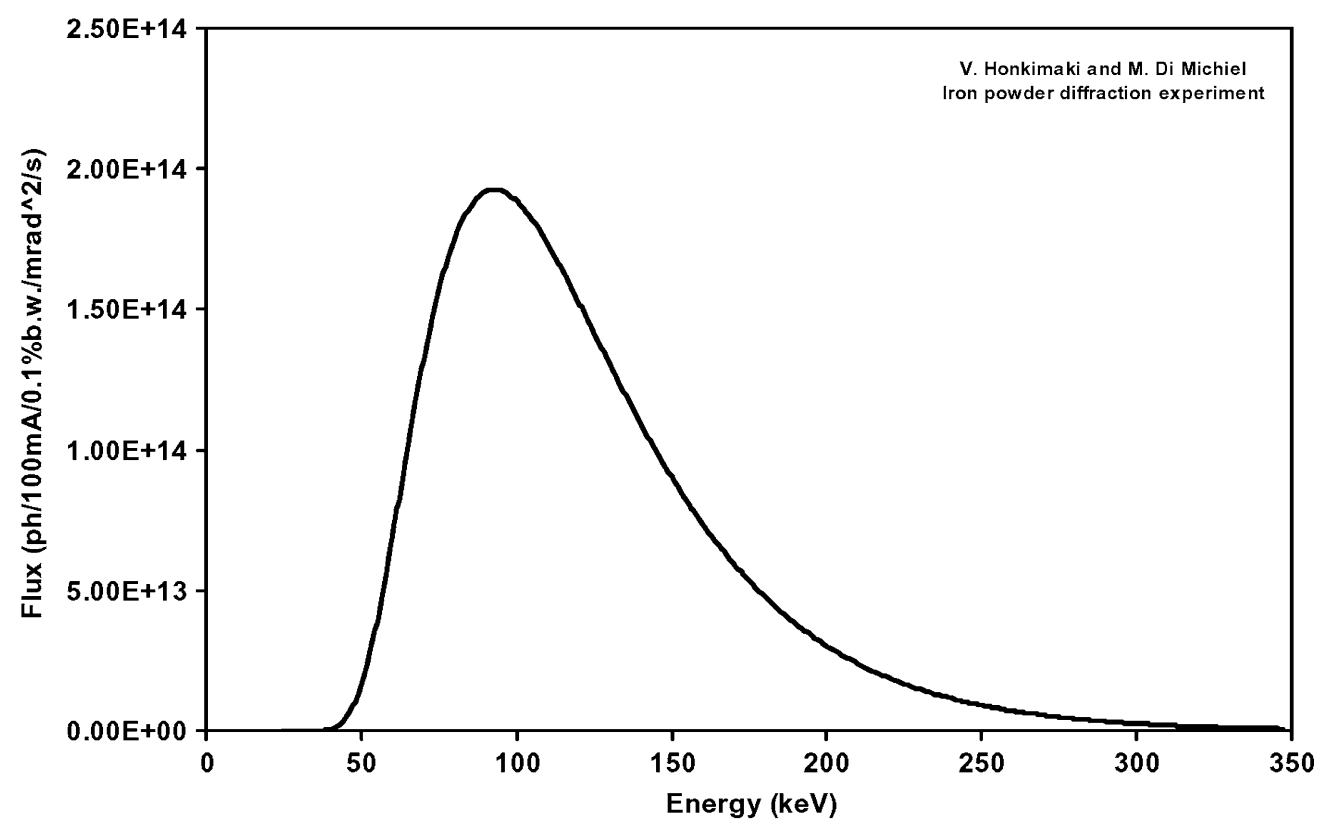

Fig. 3. Spectrum of photons on MRT facility at ESRF incident on a PMMA phantom.

PENELOPE considers the effect of binding energies of electrons and the Doppler effect on the scattered photons. For X-ray energies far above any absorption edge, the effect of the electron binding energy and a Doppler shift in scattered photon energy is limited. Due to the fact that the photons, for X-ray energies used in MRT, undergo several Compton collisions before being absorbed these corrections might be of some importance. The photons are simulated in a detailed manner, collision by collision down to absorption.

The electron interactions of importance in the MRT simulations are elastic scattering and electron impact ionization. The elastic scattering of low-energy electrons generated in the Compton interactions is of particular importance since it determines how far into the valley region the electrons are transported. For elastic electron scattering PENELOPE uses a modified Wentzel distribution with parameters obtained from relativistic partial-wave analysis. For electron impact ionization a generalized oscillator strength model is used. This model has been shown to give stopping power values in good agreement with data tabulated in ICRU 37 [16].

A mixed simulation scheme for electrons is implemented in the code, according to established terminology, where electron interactions with large energy losses and large angular deflections (both inelastic and elastic) are simulated collision by collision and small angular deflections and energy losses are treated in a grouped manner. The user-defined parameters $(\mathrm{C} 1, \mathrm{C} 2, \mathrm{WCC})$ in the mixed simulation algorithm can be set so that the simulation is run along a scale from purely detailed simulation collision by collision to grouping of many electron collisions along a single MC step. For the simulations done in this work the detailed mode was used inside and close to the sensitive volume. This was possible since the generated secondary electrons are of relatively low energy and therefore they suffer comparatively few collisions before being absorbed.

In order to achieve accuracy in the simulations, all cut-off values were kept reasonably low inside and in the close vicinity of the sensitive volume; $1 \mathrm{keV}$ for both photons and electrons. Then the residual range of an absorbed particle will be shorter than the length to traverse the smallest volume used $(1 \mu \mathrm{m})$. Further away from the sensitive volume the cut-off energies for electrons were considerably higher. This increased the speed of the simulations.

The sensitive volume of the MOSFET is very small compared to the PMMA-phantom in which it is positioned. Many more interactions will take place in the phantom than in the sensitive oxide volume. In order to achieve a better precision (smaller variance) of the estimated dose in the sensitive volume, a statistical variance reduction method called splitting was used. This method consists of multiplying particles moving in regions of interest by a constant factor and at the same time reducing their statistical weight by some amount $t$. The estimated dose and variance, for a given number of primary photon histories is then

$$
\begin{aligned}
D & =\sum_{i=1}^{N} w_{i} \cdot d_{i} \\
\sigma_{D}^{2} & =\sum_{i=1}^{N}\left(w_{i} \cdot d_{i}\right)^{2}-D^{2}
\end{aligned}
$$

where $N$ is the number of interactions in the sensitive volume, $w_{i}$ is the weight associated with the interacting particle and $d_{i}$ is the dose contribution in interaction $i$.

A total of $10^{9}$ primary photon histories were simulated. The run took about 8 hours on a Pentium 4 computer.

\section{E. GEANT4 Monte Carlo Code}

The GEANT4 Monte Carlo toolkit simulates the interaction of fundamental particles with matter [17]. It is based on Object Oriented programming and is composed of a collection of $\mathrm{C}++$ classes, each representing a particular aspect of the simulations process (geometry, tracking, visualization, etc.). 
Among these packages is the Low Energy Electromagnetic package that provides implementations of physics processes for electrons, photons, charged hadrons and ions, extended down to lower energies than those included in the so-called standard Geant 4 package. The electromagnetic processes included in the package are the Photoelectric, Compton and Rayleigh effects for photons, and bremsstrahlung and ionization for electrons. Integrated cross sections from the Lawrence Livermore database are used to compute the mean free path of each process.

For photons, the Compton sampling method is based on the product of the Klein-Nishina formula and the scattering functions contained in the Evaluated Photon Data Library (EPDL97) [18]. The scattering functions permit consideration of atomic effects for electrons, which become increasingly important at low energies. The sampling method for the Rayleigh effect is based on the product of the Rayleigh formula multiplied by form factors contained in the EPDL97. The sampling method for the photoeffect relies on choosing the first ionized subshell according to the subshell cross sections.

For electrons, energy spectra of radiated photons contained in the Evaluated Electron Data Library (EEDL) [19] are used to sample bremsstrahlung photon energy. A simplified angular distribution based on the cross section formula by Tsai is used to sample the angle of the photons with respect to the parent electron momentum. For ionization, the sampling method is based on energy spectra of delta rays contained in the EEDL, and the process is treated as a number of discrete interactions.

No cuts were implemented for the GEANT4 simulations and secondary particles were produced down to the energetic limitations of the respective physics models, namely, $1 \mathrm{eV}$ for the Rayleigh and Compton effects, down to the lowest binding energy for each element for the photoelectric effect, down to 10 $\mathrm{eV}$ for bremsstrahlung, and down to the lowest subshell binding energy for each element for electron ionization. $10^{9}$ primary photons were simulated. Owing to the long computation times, the simulation was ported to the 304 processor Dell Linux Pentium 4 Beowulf cluster located at the Australian Centre for Advanced Computing and Communications [20]. Using ten nodes, the time required for each simulation was approximately 24 hours.

\section{F. Irradiation Conditions for EO MOSFET}

The irradiation conditions for the EO MOSFET were standard MRT conditions except that the multislit collimator was replaced by a single vertical tungsten slit. The vertical tungsten slit was mounted on a computer-controlled stage with pitch, yaw and roll positioning capability with an angular accuracy of 0.001 radians. The vertical slit width could be reduced down to $5 \mu \mathrm{m}$ so as to produce a single microbeam of similar width. The alignment of the slit with respect to the X-ray beam direction was carefully checked independently to ensure minimal and symmetric scatter from the two sides of the vertical slit. The height of the single microbeam was determined by a similar slit oriented at $90^{\circ}$ to the vertical slit i.e., a horizontal slit. In the experiments described here the width and height of the microbeam was 25 and $500 \mu \mathrm{m}$ respectively.

The TOT 500 K-type MOSFET detector was used in the EO mode for successive measurements with a step size of $5 \mu \mathrm{m}$,

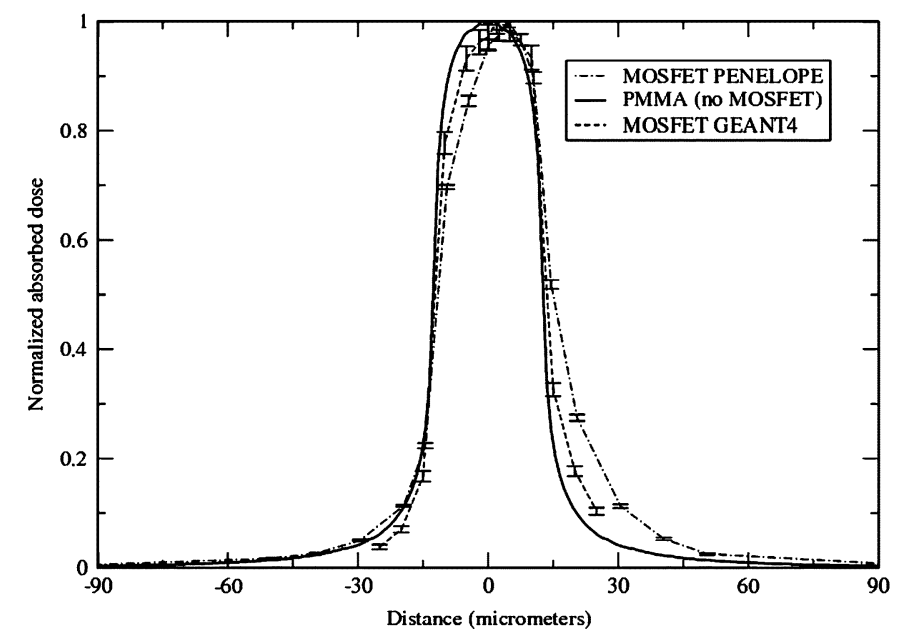

Fig. 4. Comparison of the simulated dose profiles for a PMMA phantom (solid line) and EO MOSFET (dashed and dotted-dashed lines). Error bars for PMMA simulation omitted for clarity (similar to errors for dotted-dashed line).

$+15 \mathrm{~V}$ of gate bias was applied during each exposure. The exposure time was independently measured by a radiation-shielded photodiode connected to an oscilloscope at the end of the beam path behind the PMMA phantom. It should be noted that variations of order $3 \%$ in the exposure time as measured by the photodiode could also be observed in the response of the EO MOSFET detector.

The radiation induced voltage shift $(\Delta \mathrm{V})$ at the peak position was $200 \mathrm{mV}$ with the irradiation exposure time of $29.97 \mathrm{mil}-$ liseconds. The exposure time was increased once $\Delta \mathrm{V}$ reduced to levels below $100 \mathrm{mV}$ in order to ensure the uncertainty in any one measurement was less than $1 \%$. Such voltage shifts also ensure that, starting with a fresh MOSFET, there is no danger of degradation of the linearity of MOSFET shift during a scan sequence. Such degradation could give rise to a similar distortion of the curve.

\section{RESUltS AND Discussion: SimUlation}

\section{A. Single Microbeam}

Simulations were carried out with the PENELOPE code for dose deposited in PMMA without an EO MOSFET and with the MOSFET in a PMMA phantom at depth $7 \mathrm{~cm}$. Simulations were carried out using the GEANT4 toolkit for the case of the EO MOSFET in PMMA. Fig. 4 shows the results, with a reduced dose on the left-hand part of the gate dose deposition. The penumbra bellow $70 \%$ of dose peak on the left side of the dose deposited in $\mathrm{SiO}_{2}$ is in good agreement with dose deposited in PMMA ( $Z$ of PMMA and epoxy are much less different than $\mathrm{Si}$ and epoxy). The lower penumbral dose calculated by the GEANT4 simulation may be related to the inaccuracy of the condensed history approach used in the model of electron scatter interactions. These interesting phenomena are related to not only the interfaces of two materials of different $\mathrm{Z}$ but also result from the small size of the radiation field in comparison with the detector components (body and epoxy globule).

The direction of the EO MOSFET scan, relative to stationary single microbeam, is shown in Fig. 2. The dose profile of Fig. 4 clearly shows asymmetry, a shift in the maximum dose point of 


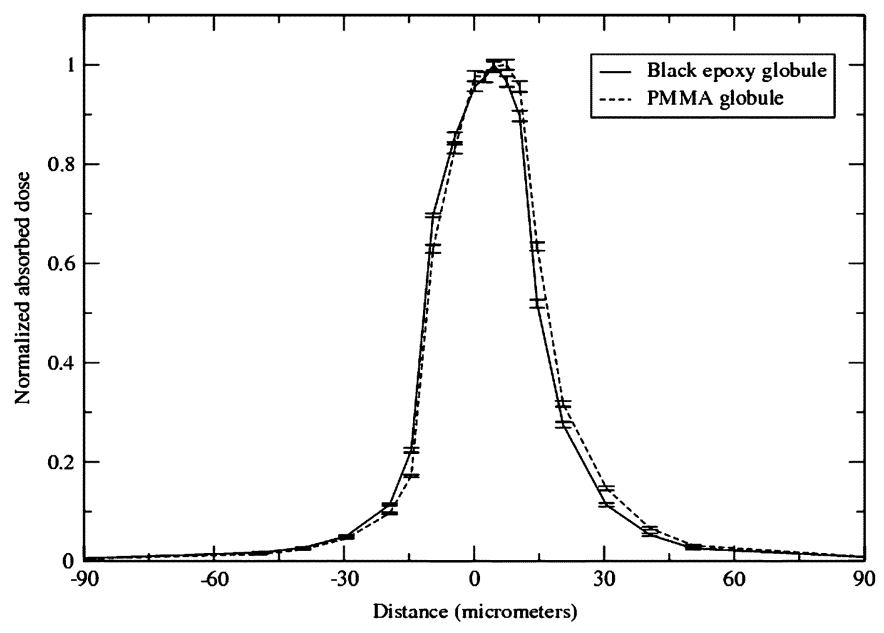

Fig. 5. Comparison of dose profile of bare EO MOSFET (i.e., PMMA globule) and EO MOSFET with black epoxy (PENELOPE code).

the dose profile is obtained for the EO MOSFET. By contrast, the dose profile at depth $7 \mathrm{~cm}$ in a PMMA phantom is symmetrical. The shift to the right of about $5 \mu \mathrm{m}$ is therefore to be explained as related to DEE arising from laterally scattered electrons from the $\mathrm{Si}$ body entering the $\mathrm{SiO}_{2}$. There is a lack of laterally scattered electrons from the epoxy arising from poor electronic equilibrium. When the $\mathrm{SiO}_{2}$ gate oxide coincides with the beam axis, we are therefore not at the point of maximum dose deposited in the oxide, as expected. The number of electrons scattered from the silicon body is larger than from the epoxy side - a result of the higher $Z$ value of $\mathrm{Si}(Z=14)$ versus that of black epoxy "filled" with silica $(Z \sim 7)$. DEE grows faster than the number of photons in the microbeam when the larger part of the microbeam is crossing the Si body; thus, the maximum dose in the oxide shifts to the right relative to the beam axis.

A comparison of the range of electrons in silicon and the photon spectrum helps to understand this. For $100 \mathrm{keV}$ electrons the range in silicon is about $80 \mu \mathrm{m}$ and for $200 \mathrm{keV}$ is $250 \mu \mathrm{m}$; the range of the electrons is larger than the width of a microbeam. This allows them to produce dose enhancement in the oxide. Even when the microbeam lies within the Si body, the scattered electrons produce a tail in the right-hand part of the dose response curve. On the other hand, the number of electrons scattered from the epoxy in the direction of the gate is less due to the low $\mathrm{Z}$ of the epoxy. The range of electrons generated by our MRT photons is larger than the beam width $(25 \mu \mathrm{m})$, again leading to lateral DEE. Moving the beam to the right relative to the MOSFET detector increases the radiation field on the epoxy side. Increasing scattering of electrons in the gate direction does not compensate the decreasing rate of scattered electrons from the Si body in the gate direction, again due to lateral disequilibrium. It is interesting that longitudinal charge particle equilibrium does exist and does not change with depth for the well-aligned EO MOSFET, allowing correct measurements of the depth dose profile using Bragg Gray cavity theory.

To understand the effect of the epoxy globule on the distortion of the dose profile, we compared the dose profile simulated

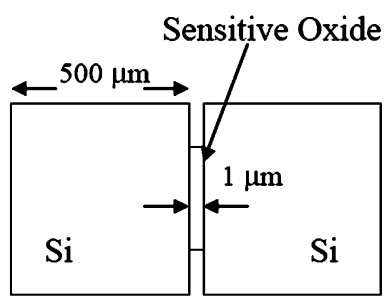

Fig. 6. Geometry of the proposed EOFF MOSFET.

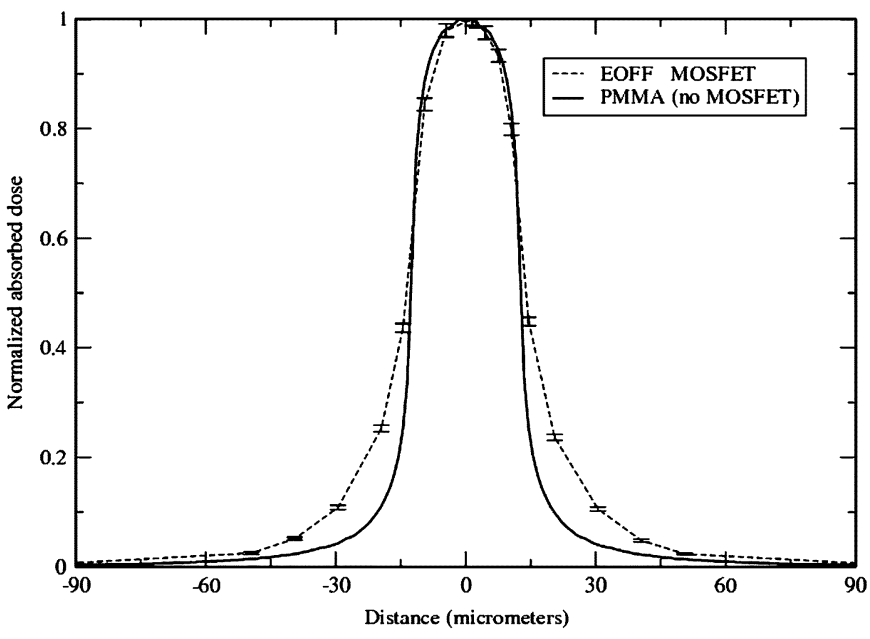

Fig. 7. Comparison of simulated dose profiles for dose in PMMA and EOFF MOSFET (PENELOPE).

with the EO MOSFET with a PMMA globule of the same shape. Fig. 5 shows that the black epoxy is very close to PMMA in terms of the lateral electron scattering properties on this photon spectrum. A small increase of the dose on the right side of the microbeam in comparison with epoxy case suggests that the black epoxy has a slightly higher cross section than PMMA.

To compensate for the asymmetry, an EOFF method is proposed (shown in Fig. 6). Simulation results comparing the dose profile measured with the EOFF MOSFET to that in PMMA are shown in Fig. 7. The EOFF MOSFET has excellent agreement with the upper part of PMMA dose profile, and both have an FWHM of $25 \mu \mathrm{m}$ and are symmetrical. The larger tailing from both sides of the dose profile within $25-50 \mu \mathrm{m}$ laterally is a result of large amount of electrons scattered from $500 \mu \mathrm{m}$ Si body in comparison with PMMA when the microbeam is totally within the Si body. This effect can be contributing to the increase of dose measured in the valley in the case of multiple beams, leading to a decreased value of the peak-to-valley dose ratio (PVDR) in comparison with MC simulations for the microbeam beam array.

\section{B. Peak-to-Valley Ratio in Multi-Microbeam MRT}

The phenomena which we have described may affect the measurement of an important parameter: the PVDR [6]. Fig. 8 shows a typical MRT microbeam array with a pitch of $200 \mu \mathrm{m}$ and a beam width of $25 \mu \mathrm{m}$. The dose in the valley (central region between two peaks) is determined by the superposition of scattered photons from neighboring microbeams. Fig. 8(a) and (b) shows an experimental arrangement for measurement 


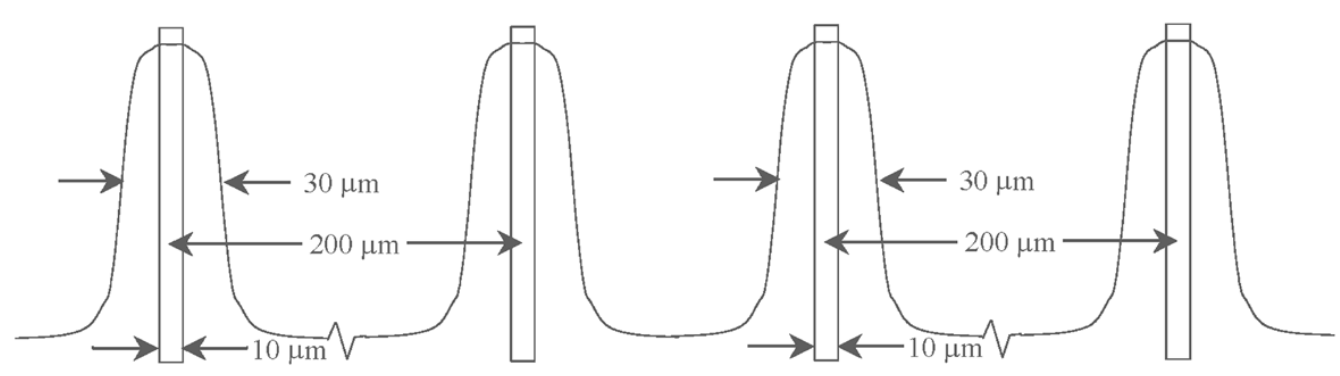

a)
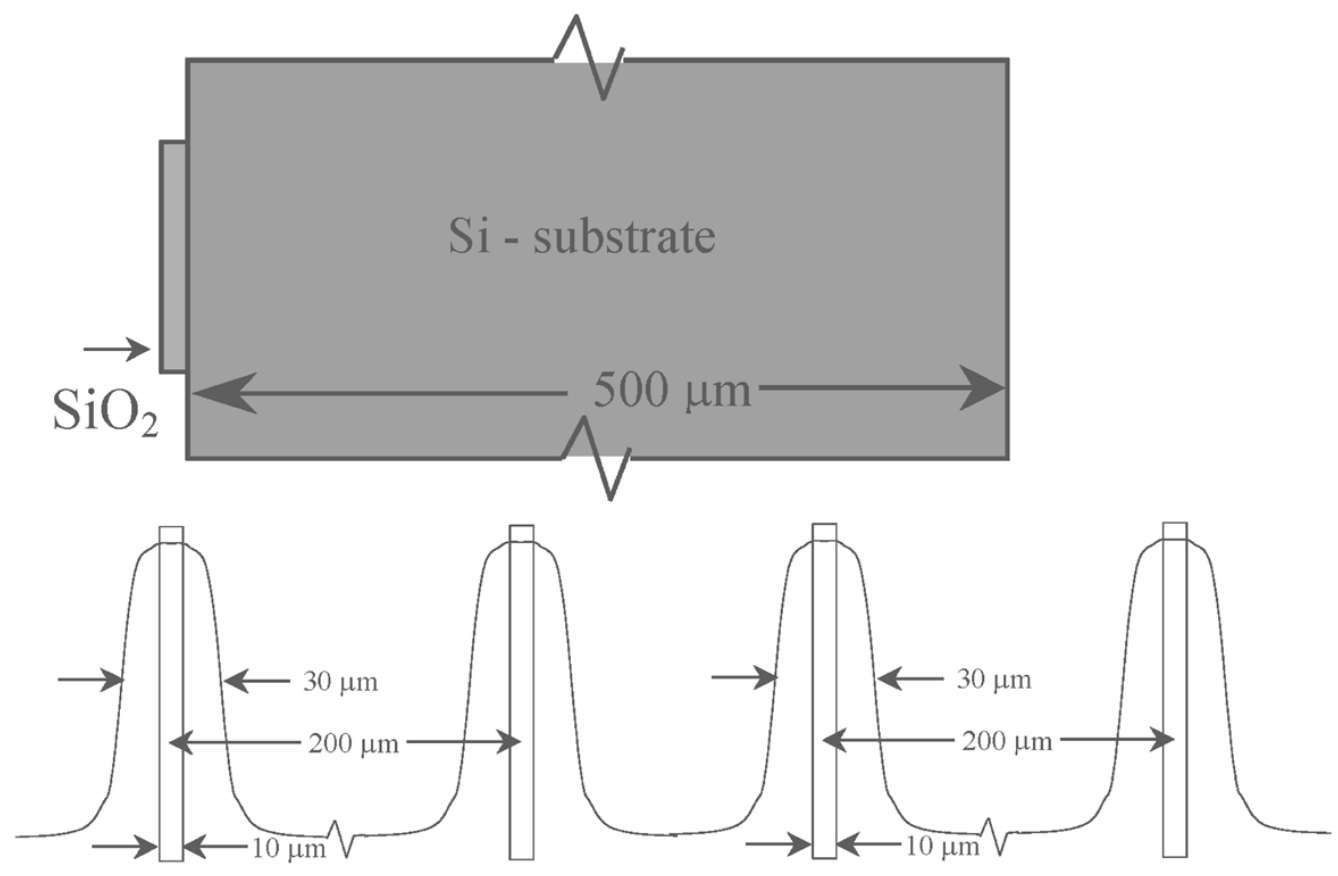

b)

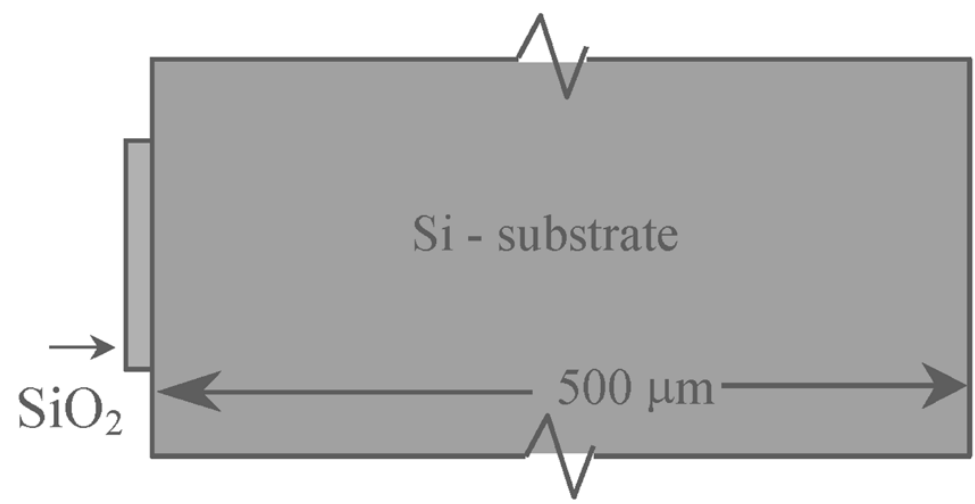

Fig. 8. $\mathrm{SiO} 2$ sensitive layers placed $\mathrm{EO}$ in a microbeam array at (a) peak dose and (b) at valley.

using the edge-on MOSFET technique. The doses in the peak and valley are enhanced by electrons due to the interaction of the right-hand neighboring beam with the Si body. One can see that the doses added to a peak by scattering from two adjacent 2 peaks [Fig. 8(a)] are less than the dose increments in the case of valley dose measurements. In this case, 2.5 peaks [Fig. 8(b)] contribute to the dose in the gate oxide originating in the scattered electrons from the Si body which is more essential due to low dose in a valley. The first two adjacent microbeams are closer to the $\mathrm{Si}-\mathrm{SiO}_{2}$ interface by $100 \mu \mathrm{m}$ in case b) then in case a). These phenomena can produce relatively high dose increments to the valley dose, reducing the experimental PVDR in comparison with the simulated PVDR. A thinner Si body will dramatically improve valley dose measurement. It is obvious that the absolute values of these dose increments will depend on the spectra of the photons.

\section{RESULTS AND DISCUSSION: EXPERIMENT}

Fig. 9 shows the scan of a single microbeam with a 5 micron step. The asymmetry of the dose profile is clearly observed. This is related to the beam profile itself or the lateral DEE as explained in Section III. The Monte Carlo results indicate that the real center of the microbeam occurs at the position defined by the middle of the two positions with 50\% of the maximum in the normalized dose curve perturbed by the MOSFET (MOSFET PENELOPE curve in Fig. 4). From Fig. 4 the shift in the peak of the unperturbed and perturbed curves is $7.5 \pm 2 \mu \mathrm{m}$ in the 


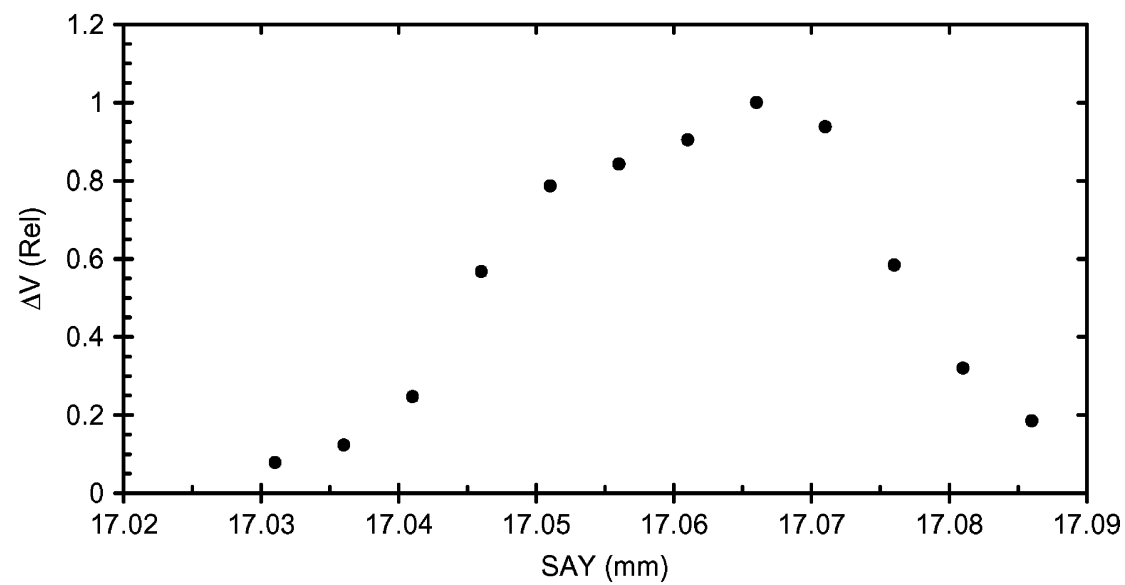

Fig. 9. Scan of a single microbeam with a nominal FWHM width of 25 microns using a MOSFET. The step size is 5 microns. The deduced FWHM of the microbeam from the scan is $35+2$ microns. Error in positioning is less than $0.2 \mathrm{~mm}$ for the $x-y$ stage used.

positive direction. Similarly, we can deduce the real center of the microbeam in the experimentally measured figure from the middle of the two positions with $50 \%$ of the maximum in the normalized response curve in Fig. 9. In this case the shift in the peak of the unperturbed and perturbed curves is $5+2.5 \mu \mathrm{m}$ in the positive direction, which seems in reasonable agreement with the Monte Carlo.

\section{CONCLUSION}

We have shown that a MOSFET detector, scanned through a microbeam edge-on (EO), is a unique tool for synchrotron microbeam dosimetry. The oxide, one micrometer or less in thickness, presents to the beam the smallest cross section known in dosimetry. However, we must then deal with the lateral dose enhancement related to the different impact of the microbeam on elements of the detector setup having different average Z. With a single MOSFET, this produces an artificial distortion of the measured dose profile. This mechanism has been confirmed by PENELOPE and GEANT4 simulations at two research centers and also by experimental results obtained at the MRT facility single beam at ESRF, Grenoble, France. Closer simulation and experiments with a more accurate design of MOSFET will allow us to understand and reduce the asymmetry error in question. As well as the straightforward techniques of optimizing the encapsulations, we are studying two more advanced techniques. The proposed edge-on face-to-face (EOFF) MOSFET detector, a die arrangement proposed here for the first time, is a logical solution to restore symmetry to radiation scattering. Further improvement will be obtained by thinning the silicon bodies of the MOSFETs.

\section{ACKNOWLEDGMENT}

The authors would like to thank the workshop at the ESRF and the Australian Centre for Advanced Computing and Communications for providing access to the Barossa cluster used for the GEANT4 simulations.

\section{REFERENCES}

[1] W. J. Poch and A. G. Holmes-Siedle, "The mosimeter-A new instrument for measuring radiation dose," RCA Engineer, vol. 16, no. 3, pp. 56-59, Oct./Nov. 1970.
[2] J. L. Autran, P. Masson, N. Freud, C. Raynaud, and C. Rickel, "Microirradiation experiments in MOS transistors using synchrotron radiation," IEEE Trans. Nucl. Sci., vol. 47, no. 3, pp. 574-579, Jun. 2000.

[3] A. G. Holmes-Siedle and L. Adams, "RADFETs: A review of the use of metal-oxide-silicon devices as integrating dosimeters," Int. J. Radiat. Phys. Chem., vol. 28, no. 2, pp. 235-244, 1986.

[4] A. G. Holmes-Siedle and L. Adams, Handbook of Radiation Effects. London, U.K.: Oxford Univ. Press, 2002, ISBN 0-19-850 733-X

[5] G. Kaplan, A. B. Rosenfeld, B. Allen, J. T. Booth, M. G. Carolan, and A. Holmes-Siedle, "Improved spatial resolution by MOSFET dosimetery of an X-ray microbeam," Med. Phys., vol. 27, no. 1, pp. 239-244, 2000.

[6] A. Rosenfeld, G. Kaplan, B. Allen, F. A. Dilmanian, T. Kron, and A. Holmes-Siedle, "MOSFET dosimetry of X-ray microbeams," IEEE Trans. Nucl Sci., vol. 46, no. 6, pp. 1774-1780, Dec. 1999.

[7] A. B.Rosenfeld, M. L. F. Lerch, T. Kron, E. Brauer-Krisch, A. Bravin, A. Holmes-Siedle, and B. J. Allen, "Feasibility study of on-line, high spatial resolution MOSFET dosimetry in static and pulsed X-ray radiation fields," IEEE Trans. Nucl. Sci., vol. 48, no. 6, pp. 2061-2068, Dec. 2001.

[8] E. Brauer-Krisch, A. Bravin, M. Lerch, A. B. Rosenfeld, J. Stepanek, M. DiMichiel, and J. A. Laissue, "MOSFET dosimetry for microbeam radiation therapy at the European synchrotron radiation facility," Med. Phys., vol. 30, no. 4, pp. 583-589, 2003.

[9] C. M. Dozier and D. B. Brown, "Effect of photon energy on the response of MOSFET devices," IEEE Trans. Nucl. Sci., vol. 28, no. 6, pp. 4137-4141, Dec. 1981.

[10] D. M. Long, D. G. Millward, and J. Walalce, "Dose enhancement effect in semiconductor devices," IEEE Trans. Nucl. Sci., vol. 29, no. 6, pp. 1980-1984, Dec. 1982.

[11] R. N. Hamm, "Dose calculations for $\mathrm{Si}-\mathrm{SiO} 2$ layred structures by $\mathrm{X}$ rays and Co-60 gamma rays," IEEE Trans. Nucl. Sci., vol. 33, pp. 1236-1239, 1986.

[12] D. M. Fleetwood, P. S Winokur, L. J. Lorence, W. Beezhold, P. V. Dressendorfer, and J. R. Schwank, "The response of MOS devices to dose-enhanced low energy radiation," IEEE Trans. Nucl. Sci., vol. 33, pp. 1245-1251, 1986.

[13] G. J. Brucker, S. Kroneneberg, and F. Gentner, "Effect of package geometry, materials and die design on energy dependence of pMOS dosimeters," IEEE Trans. Nucl. Sci., vol. 42, no. 1, pp. 33-40, Feb. 1995.

[14] A. B. Rosenfeld, M. G. Carolan, G. Kaplan, B. J. Allen, and V. I. Kivrich, "MOSFET Dosimeters: Role of encapsulation in mixed gamma-neutron and megavoltage X-ray fields," IEEE Trans. Nucl. Sci., vol. 42, no. 6 , pp. 1870-1877, Dec. 1995.

[15] B. Wang, C. H. Kim, and X. G. Xu, "Monte Carlo modeling of a high sensitivity MOSFET dosimeter for low and medium energy photon sources," Med. Phys., vol. 31, no. 5, pp. 1003-1008, 2004.

[16] F. Salvat, J. M. Fernández-Varea, and J. Sempau, "PENELOPE, a code system for Monte Carlo simulation of electron and photon transport," presented at the Workshop/Training Course, Jul. 7-10, 2003.

[17] S. Agostinelli et al., "GEANT4: A simulation toolkit," Nucl. Instrum. Methods Phys. Res. A, pp. 250-303, 2003.

[18] D. Cullen et al., EPDL97: The Evaluated Photon Data Library, vol. 6, 1997. 97 version, UCRL-50400, Rev. 5

[19] S. T. Perkins et al., Tables and Graphs of Electron Interaction Cross Sections from $10 \mathrm{eV}$ to $100 \mathrm{GeV}$ Derived from the LLNL Evaluated Electron Data Library (EEDL), UCRL-50 400, vol. 31, 1997.

[20] Australian Centre for Advanced Computing and Communications (2005). [Online]. Available: http://www.ac3.edu.au/edu/ 